Applied Scientific Research 39: 45--53 (1982) 0003-6994/82/010045-9 \$00.20/0

(c) 1982 Martinus Nijhoff Publishers, The Hague. Printed in the Netherlands.

\title{
Ground water movement due to arbitrary changes in water level
}

\author{
R. SESHADRI* and T.Y. NA**
}

* Senior Mechanical Engineer, Syncrude Canada Ltd., Edmonton, Canada

** Professor, Department of Mechanical Engineering, University of Michigan - Dearborn, Michigan, USA

\begin{abstract}
The non self-similar boundary value problem of ground water movement due to arbitrary changes in water level is solved. The non self-similar solutions are generated from known similarity solutions using numerical methods.
\end{abstract}

\section{Nomenclature}

$K$ permeability of the aquifer

$h$ height of water level above the impermeable surface

$V$ void ratio

$x$ space coordinate

$t$ time

$\bar{x}$

$\bar{t}$ non-dimensional variables

$\bar{h}$

$L$ characteristic length

$f$ dependent variable, defined in equation (5)

$q$ flow through a unit width, defined in equation (23a)

Greek letters

$\zeta$ similarity variable

$\tau$ time coordinate in non self-similar description

Subscripts

$n$ refers to the value of the function at time $\tau$

Superscripts

$\nu$ is the number of the iteration

\section{Introduction}

The problem of ground water movement due to an arbitrary change in water level is considered in this paper. The boundary-value problem that results is non self-similar [3], and therefore numerical methods must be applied to 


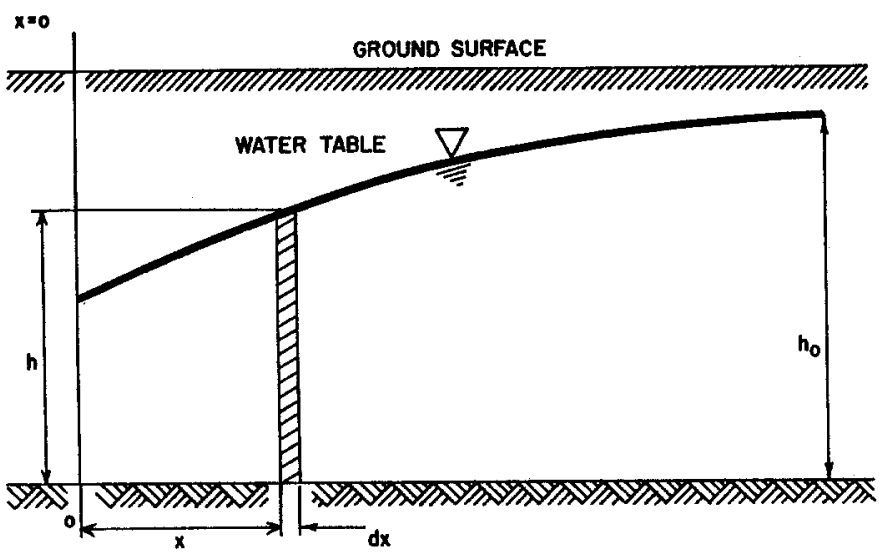

Figure 1. Ground water flow

obtain the solution. A scheme that has been successfully applied to boundary layer theory [4], is used here.

The equation governing the ground water movement which uses the Dupuit-Forchheimer idealization [1] can be written as:

$$
K \frac{\partial}{\partial x}\left(h \frac{\partial h}{\partial x}\right)=V \frac{\partial h}{\partial t},
$$

where $K$ is the permeability of the homogeneous and isotropic aquifer, $V$ is the void ratio, $h$ is the height of the water table above the impermeable surface, $x$ is the space coordinate and $t$ is time. It is assumed that all the flow takes place below the water table and that the aquifer rests upon an impermeable horizontal bed (Figure 1). The auxiliary conditions for the problem can be written as:

and

$$
\begin{aligned}
& h(x, 0)=h_{0}, \\
& h(x \rightarrow \infty, t)=h_{0},
\end{aligned}
$$

$$
h(0, t)=H(t), \quad t>0
$$

where $H(t)$ is an arbitrary variation of the water level at $x=0$.

\section{Transformation of the differential equations}

The governing differential equation derived in the preceding section can be written in a non-dimensional form as follows, by introducing:

$$
\bar{x}=\frac{x}{L} ; \quad \bar{t}=\frac{K h_{0} t}{L^{2}} ; \quad \beta(t)=\frac{H(t)}{h_{0}} ; \quad \bar{h}(\bar{x}, \bar{t})=\frac{h(x, t)}{h_{0}},
$$


where $L$ is the characteristic length.

Equations (1) and (2) then become:

$$
\begin{aligned}
& \frac{\partial}{\partial \bar{x}}\left(\bar{h} \frac{\partial \bar{h}}{\partial \bar{x}}\right)=\frac{\partial \bar{h}}{\partial \overline{\bar{t}}}, \\
& \bar{h}(\bar{x}, 0)=1, \\
& \bar{h}(\infty, \bar{t})=1,
\end{aligned}
$$

and

$$
\bar{h}(0, \bar{t})=\beta(\bar{t}) .
$$

Equation (4c) prevents invariance under a one-parameter transformation group that would have led to a similarity solution [3]. However, it will be shown that equations (3) and (4) can be transformed to a special form which is very convenient for solution by the finite difference method. The necessary transformation has to be derived by a procedure similar to the derivation of a similarity transformation. The key step is to ignore the source of the nonsimilarity which, for this example, is equation (4c). A similarity analysis can then be applied to the problem which excludes the non-similar term. For the remaining problem description under consideration, such an analysis would result in the similarity transformation:

$$
\zeta=\frac{\bar{x}}{\sqrt{\bar{t}}} ; \quad f(\zeta)=\bar{h}(\bar{x}, \bar{t}) .
$$

The transformation needed for the complete problem (including the source of non-similarity) can then be written as follows:

$$
\tau=\bar{t}, \quad \zeta=\frac{\bar{x}}{\sqrt{\bar{t}}}, \quad f(\zeta, \tau)=\bar{h}(\bar{x}, \bar{t}) .
$$

In other words, the number of independent variables is not decreased. Equations (3) and (4) are now transformed to the following form:

$$
f \frac{\partial^{2} f}{\partial \zeta^{2}}+\left(\frac{\partial f}{\partial \zeta}\right)^{2}+\frac{1}{2} \zeta \frac{\partial f}{\partial \zeta}=\tau \frac{\partial f}{\partial \tau}
$$

subject to the boundary conditions:

$$
\begin{aligned}
& f(0, \tau)=\beta(\tau), \\
& f(\infty, \tau)=f(\infty, 0)=1 .
\end{aligned}
$$

Equations transformed into the form of equation (7) have been widely used in the boundary layer theory [4]. There are three advantages over other possible forms. The primary advantage is that the starting process is very simple. At $\tau=0$ all $\tau$-dependency is removed, leaving only an ordinary differential equation to be solved. The second important advantage is that 
most of the variation of the penetration depth has been removed. This property greatly simplifies the problem of solving the ordinary differential equations, as will be seen later. The third advantage of this equation is that the $\tau$-term is expressed explicitly in a sense that it represents a "correction factor' to the similar solution.

\section{Numerical solutions}

Equations (7) and (8) will now be solved by the finite difference method. As a first step, derivatives in the $\tau$-direction are replaced by finite differences. For example,

$$
\frac{\partial f}{\partial \tau}=\frac{f_{n}-f_{n-1}}{\Delta \tau} .
$$

The function, $f$, and its $\zeta$ are replaced by averages in the following manner:

$$
\begin{aligned}
\frac{1}{2}\left[\left\{f_{n} f_{n}^{\prime \prime}\right.\right. & \left.+\left(f_{n}^{\prime}\right)^{2}+\frac{1}{2} \zeta\left(f_{n}^{\prime}\right)\right\} \\
& \left.+\left\{f_{n-1} f_{n-1}^{\prime \prime}+\left(f_{n-1}^{\prime}\right)^{2}+\frac{1}{2} \zeta\left(f_{n-1}^{\prime}\right)\right\}\right] \\
& =\tau_{n-1 / 2} \cdot\left(\frac{f_{n}-f_{n-1}}{\Delta \tau}\right),
\end{aligned}
$$

where $f_{n}$ refers to $f$ at time $\tau$, and $f_{n-1}$ to time $t-\Delta \tau$.

Rewriting equation (11) after some rearrangement, we obtain:

$$
f_{n} f_{n}^{\prime \prime}+\left(f_{n}^{\prime}\right)^{2}+\frac{1}{2} \zeta f_{n}^{\prime}-\alpha_{n-1 / 2} f_{n}=R_{n-1},
$$

where

and

$$
\alpha_{n-1 / 2}=\frac{2 \tau_{n-1 / 2}}{\Delta \tau}=\frac{\tau_{n}+\tau_{n-1}}{\Delta \tau}
$$

$$
\begin{aligned}
R_{n-1}= & -\alpha_{n-1 / 2} f_{n-1}-f_{n-1} f_{n-1}^{\prime \prime} \\
& -\left(f_{n-1}^{\prime}\right)^{2}-\frac{1}{2} \zeta\left(f_{n-1}^{\prime}\right) .
\end{aligned}
$$

The right-hand side of equation (11a) is known in the recursion scheme above. By letting $n=0,1,2, \ldots$, etc., in equation (11a), a sequence of equations for the solution of $f_{0}, f_{1}, f_{2}, f_{3}, \ldots$, etc., and their derivatives can be obtained.

Equation (11a) is nonlinear. However, it can be linearized by the method of quasi-linearization [5], and can therefore be written as

$$
F\left(f_{n}, f_{n}^{\prime}, f_{n}^{\prime \prime}, \zeta\right)=0 .
$$

For the $\nu$ th iteration, we can write equation (12) as

$$
F\left(f_{n}^{(\nu)},\left(f_{n}^{\prime}\right)^{(\nu)},\left(f_{n}^{\prime \prime}\right)^{(\nu)}, \zeta\right)=0 .
$$


For the $(\nu+1)$ th iteration, we have

$$
\begin{aligned}
F\left\{f_{n}^{(\nu+1)},\left(f_{n}^{\prime}\right)^{(\nu+1)},\left(f_{n}^{\prime \prime}\right)^{(\nu+1)}, \zeta\right\} \\
=F^{(\nu)}+\left(\frac{\partial F}{\partial f_{n}^{\prime \prime}}\right)^{(\nu)}\left\{\left(f_{n}^{\prime \prime}\right)^{(\nu+1)}-\left(f_{n}^{\prime \prime}\right)^{(\nu)}\right\} \\
+\left(\frac{\partial F}{\partial f_{n}^{\prime}}\right)^{(\nu)}\left\{\left(f_{n}^{\prime}\right)^{(\nu+1)}-\left(f_{n}^{\prime}\right)^{(\nu)}\right\} \\
\quad+\left(\frac{\partial F}{\partial f_{n}}\right)^{(\nu)}\left\{\left(f_{n}\right)^{(\nu+1)}-\left(f_{n}\right)^{(\nu)}\right\}+\ldots=0 .
\end{aligned}
$$

Substituting equation (11a) into (14), the following can be obtained:

$$
\begin{aligned}
& \left(f_{n}^{(\nu)}\right)\left\{\left(f_{n}^{(\nu+1)}\right)^{\prime \prime}-\left(f_{n}^{(\nu)}\right)^{\prime \prime}\right\} \\
& \quad+\left(f_{n}^{(\nu)}\right)^{\prime \prime}\left\{\left(f_{n}^{(\nu+1)}-f_{n}^{(\nu)}\right)\right\} \\
& \quad+2\left(f_{n}^{(\nu)}\right)^{\prime}\left\{\left(f_{n}^{(\nu+1)}\right)^{\prime}-\left(f_{n}^{(\nu)}\right)^{\prime}\right\} \\
& \quad+\left(\frac{1}{2} \zeta\right)\left\{\left(f_{n}^{(\nu+1)}\right)^{\prime}-\left(f_{n}^{(\nu)}\right)^{\prime}\right\} \\
& \quad-\left(\alpha_{n-1 / 2}\right)\left\{\left(f_{n}^{(\nu+1)}\right)-\left(f_{n}^{(\nu)}\right)\right\}=0 .
\end{aligned}
$$

Rearranging equation (15),

where

$$
A_{0}^{(\nu)}\left\{f_{n}^{(\nu+1)}\right\}^{\prime \prime}+A_{1}^{(\nu)}\left\{f_{n}^{(\nu+1)}\right\}^{\prime}+A_{2}^{(\nu)}\left\{f_{n}^{(\nu+1)}\right\}=s^{(\nu)},
$$

$$
\begin{aligned}
A_{0}^{(\nu)} & =f_{n}^{(\nu)} \\
A_{1}^{(\nu)} & =2\left(f_{n}^{(\nu)}\right)^{\prime}+\frac{1}{2} \zeta \\
A_{2}^{(\nu)} & =\left(f_{n}^{(\nu)}\right)^{\prime \prime}-\alpha_{n-1 / 2} \\
& =\frac{R_{n-1}-\left[\left(f_{n}^{(\nu)}\right)^{\prime}\right]^{2}-\frac{1}{2} \zeta\left(f_{n}^{(\nu)}\right)^{\prime}+\alpha f_{n}^{(\nu)}-\alpha_{n-1 / 2}}{f_{n}^{(\nu)}}
\end{aligned}
$$

and

$$
s^{(\nu)}=2 R_{n-1}-\frac{1}{2} \zeta\left(f^{(\nu)}\right)^{\prime}+\alpha_{n-1 / 2} f_{n}^{(\nu)} .
$$

The superscript ' $v$ ' represents the number of iterations. By assuming a first approximation, equation (16) along with the boundary conditions

$$
f_{n}^{(\nu+1)}(\tau, 0)=\beta(\tau) ; \quad f_{n}^{(\nu+1)}(\tau, \infty)=1
$$

can be solved, and the next iteration determined. This process would continue until the solution converges. The solution at time $\tau$ is first approximated by the solution at time $\tau-\Delta \tau$.

Equation (16), subject to the boundary conditions (17), form a two-point 
boundary value problem. The method of superposition [5] used to solve the problem is described in Appendix A.

\section{Numerical examples}

\section{Case (1)}

Consider the variation of $\beta(\tau)$, (Figure 2 ),

$$
\begin{array}{ll}
\beta(\tau)=1+b \sin \left(\frac{\tau}{\tau_{0}} \cdot \frac{\pi}{2}\right), & \text { for } \tau \leqslant \tau_{0}, \\
\beta(\tau)=1+b, & \text { for } \tau>\tau_{0} .
\end{array}
$$

For this case, it can be expected that the nonsimilar solution will approach the similarity solution at large times $\tau$.

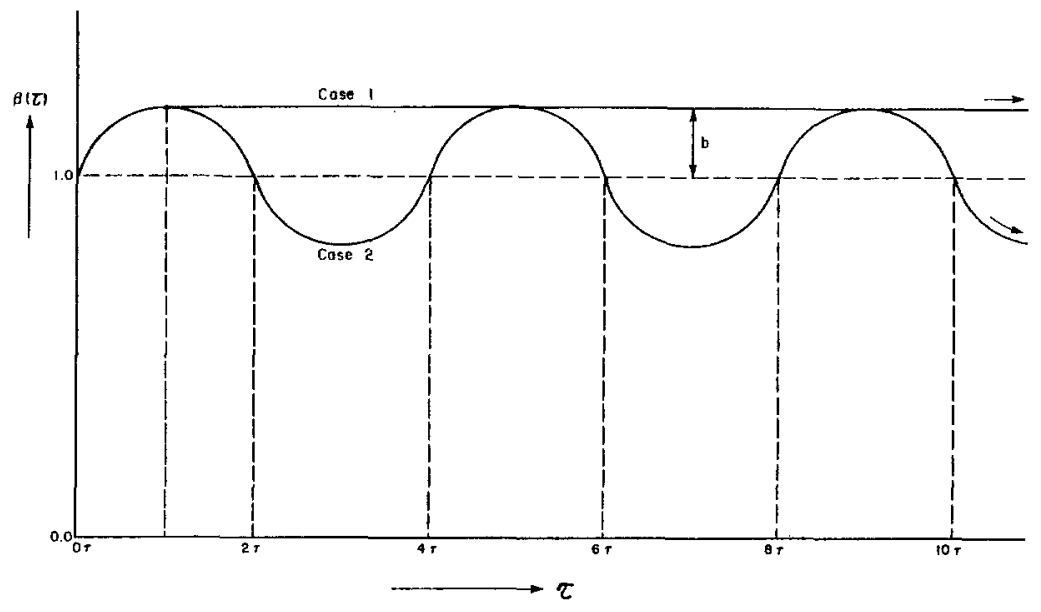

Figure 2. Variation of $\beta(\tau)$ versus $\tau$

At $\tau=0$, equation (17) becomes

$$
f f^{\prime \prime}+\left(f^{\prime}\right)^{2}+\frac{1}{2} \zeta f^{\prime}=0 .
$$

An inspection of the initial conditions shows that the solution is

$$
f_{0}(0, \zeta)=1
$$

which satisfies equation (19). Also,

$$
f_{0}^{\prime}=f_{0}^{\prime \prime}=0 \text {. }
$$

Putting $n=1$ in equation (16) and using equations (20) the 'recursion scheme' can be initiated.

For the numerical solution, $\tau_{0}=0.5 ; b=0.5,-f^{\prime}(\tau, 0)$ is plotted against 


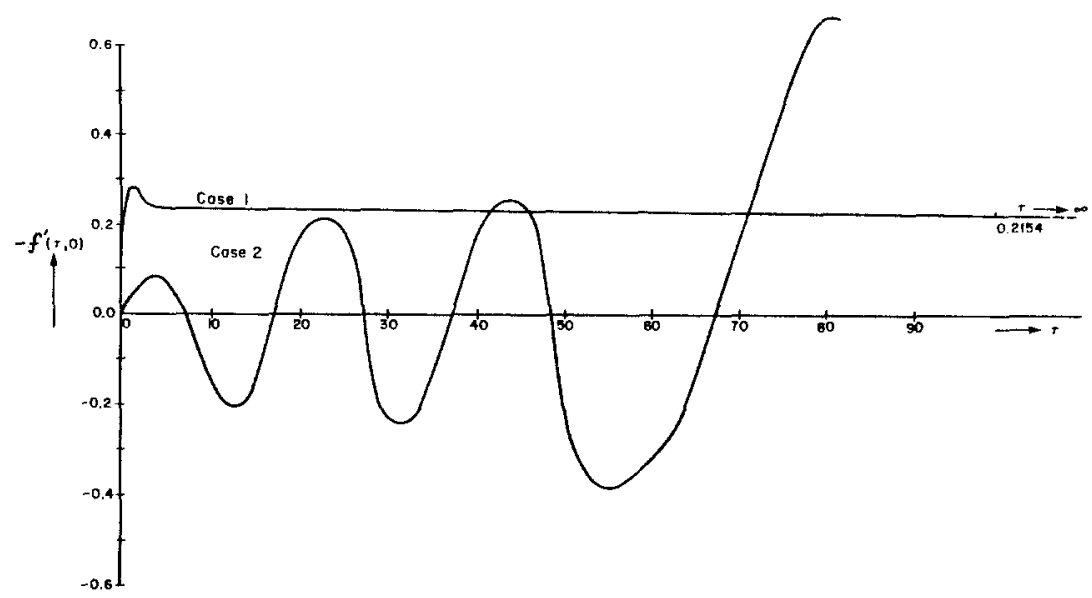

Figure 3. Plot of $-f^{\prime}(\tau, 0)$ versus $\tau$

$\tau$ (Figure 3). It can be seen that the asymptotic limit is reached when $\tau \simeq 1100$ with

$$
\begin{aligned}
& -f^{\prime}(\tau, 0)=0.2154 . \quad \text { For } \quad \tau_{0}=0.5, \quad b=1.0 \\
& \tau_{\text {lim }} \simeq 1100 \quad \text { and } \quad-f^{\prime}(\tau, 0)=0.3597 .
\end{aligned}
$$

Case 2

As a second example consider the variation

$$
\beta(\tau)=1+b \sin \left(\frac{\tau}{\tau_{0}} \cdot \frac{\pi}{2}\right) .
$$

For $b=0.1$ and $\tau_{0}=5$, the results for the non-similar problem are plotted in Figure 3.

The flow of ground water through a unit width at a distance $x$ from the origin can be expressed as

$$
q(x, t)=K h(x, t) \frac{\partial h}{\partial x} .
$$

Evaluating the flow at $x=0$ and rewriting equation (23a) in a dimensionless form,

$$
\frac{q(0, \tau)}{K h_{0}^{2} / L}=\frac{\beta(\tau)}{\sqrt{\tau}} f^{\prime}(0, \tau) .
$$

The flow can now be evaluated by using the expressions for $\beta(\tau)$, as defined in equations (18) or (22), in conjunction with Figure 3. 


\section{Conclusions}

Non-similar solutions have been generated for the non-invariant boundary value problem using known self-similar solutions. The non-similar solutions asymptotically approach similar solutions for large values of time.

From an engineering viewpoint, the ground water flow for arbitrary changes in the water level has been determined.

\section{References}

1. Bear J (1972) Dynamics of Fluids in Porous Media. American Elsevier Publishing Company.

2. Bluman GW and Cole JD (1974) Similarity Methods for Differential Equations. New York: Springer-Verlag.

3. Hansen AG (1964) Similarity Analyses of Boundary Value Problems in Engineering. Prentice Hall.

4. Keller HS (1978) Numerical Methods in Boundary Layer Theory. Annual Review of Fluid Mechanics 10:417-433.

5. Na TY (1979) Computational Methods in Engineering Boundary Value Problems. Academic Press Inc.

6. Zel'dovich YaB and Raizer YuP (1967) Physics of Shock Waves and High Temperature Hydrodynamic Phenomena. In Hayes WD and Probstein RF (eds) Vol 2. Academic Press.

\section{Appendix A}

Consider an equation of the form

$$
A_{0} f^{\prime \prime}+A_{1} f^{\prime}+A_{2} f=0,
$$

where $A_{0}, A_{1}$ and $A_{2}$ are known coefficients. The two-point boundary conditions are

$$
f(0)=b ; \quad f(\infty)=1 .
$$

To transform the two-point boundary value problem to an initial value problem, assume

$$
f=f_{1}+\mu f_{2},
$$

where $\mu$ is a constant to be determined.

Substituting equation (A.3) into (A.1) two equations are obtained.

$$
\text { (1) } A_{0} f_{1}^{\prime \prime}+A_{1} f_{1}^{\prime}+A_{2} f_{1}=0
$$

with

$$
f_{1}(0)=b
$$

and initial slope

$$
\begin{aligned}
& f_{1}^{\prime}(0)=0 . \\
& \text { (2) } A_{0} f_{2}^{\prime \prime}+A_{1} f_{2}^{\prime}+A_{2} f_{2}=0
\end{aligned}
$$

with

$$
f_{2}(0)=0
$$


and slope

$$
f_{2}^{\prime}(0)=1
$$

Therefore

$$
f^{\prime}(0)=f_{1}^{\prime}(0)+\mu f_{2}^{\prime}(0)=\mu .
$$

To determine $\mu$, the boundary condition at $\infty$ is utilized:

$$
\begin{aligned}
& f_{1}(\infty)+\mu f_{2}(\infty)=1, \\
& \mu=\frac{1-f_{1}(\infty)}{f_{2}(\infty)} .
\end{aligned}
$$

$f_{1}(\infty)$ and $f_{2}(\infty)$ can be obtained by solving equation (A.4) through (A.7). 